\section{The Relativistic Theory of the Fresnel Drag Coefficient}

Is this communication I give a somewhat more general discussion of the Fresnel drag coefficient than is usual, with the view of clarifying the relation between two expressions for it, which occur in the literature of special relativity. These expressions are normally derived separately and their relationship has recently been discussed by Synge ${ }^{1}$. I take a further step here, by showing how both results can be obtained from a single argument. This procedure directs appropriate attention to step (6) (see below), which is the crucial point in both cases. The necessary background may be outlined as follows:

Suppose a medium has velocity $v$ parallel to the $x$-axis of a reference system $S$. If light passes through the medium parallel to this direction, let its phase velooity be $u_{m}$ relative to $S$. Assume that $u_{m}=$ $c / n\left(v_{m}\right)$, where $n\left(\nu_{m}\right)$ is the refractive index of light of frequency $v_{m}$ in the medium, provided the medium is at rest in $S$ (that is, $v=0$ ). Then in the general $\operatorname{case}(v \neq 0)$ :

$$
u_{m}=c / n\left(v_{m}\right)+k v
$$

Here $k$ is a coefficient which was once supposed to measure the extent to which the rther is dragged along by the medium (Fresnel, 1818). The special relativity theory also leads to (1). For $k$, it yields the result :

$$
k=1-\frac{1}{n\left(\nu_{0}\right)^{2}}+\frac{c}{v} \frac{\nu_{0}}{n\left(\nu_{0}\right)^{r}}\left\{\frac{\mathrm{d} n(\nu)}{\mathrm{d} \nu}\right\}_{\nu=\nu_{0}}
$$

Here $r=1$ in the case of water flowing at velocity $v$ in a tube fixed in $S$, and $r=2$ for a block of glass moving with velocity $v$. These are the two cases usually described.

Consider now a uniform transparent medium in uniform motion with velocity $v$ parallel to the $x$-axis of an inertial system $S$. The medium is contained in a transparent vessel the walls of which can unfold during the motion of the medium, so that the rear face of the container (which is perpendicular to the $x$-axis) moves with a velocity $w \leqslant v$. The optical properties of the medium in the container are, however, kept homogeneous and constant in time by feeding additional material through a tube leading to the rear of the container. This means that we assume the following idealized situation: At any time $t$, contact between the rear surface of the container and the medium is about to break due to the differenee in velocity. But the gap $(v-w) \mathrm{d} t$, which one would expect to develop in time $\mathrm{d} t$, is filled in by additional material having already $v$ as its $x$-component of velocity. Contact between the rear surface and the medium is assumed to be maintained in this way ; different layers of material are involved in the contact at different instants. This situation cannot be realized exactly, but my main interest is in the two limiting cases, which can be realized.

Let the reference system in which the medium is at rest be $S^{\prime}$; that in which the rear face is at rest, be $S^{\prime \prime}$; the velocity of $S^{\prime \prime}$ as observed in $S^{\prime}$ be $w^{\prime}$; the frequency of the light when moving in vacuum and observed in $S^{\prime \prime}$ be $v_{0}^{\prime \prime}$ and the frequeney when moving in the medium and observed in $S^{\prime \prime}$ be $v_{m}{ }^{\prime \prime}$. I shall adopt this type of notation also for the frames $S$ and $S^{\prime}$ and for the phase velocity of the light, which is denoted by $u_{0}$ for vacuum and by $u_{m}$ for the medium.
By the relativistic velocity addition :

$$
u_{m}=\frac{u_{m}{ }^{\prime}+v}{1+u_{m}^{\prime} v / c^{2}}=\frac{c / n\left(\nu_{m}{ }^{\prime}\right)+v}{1+v / \operatorname{cn}\left(\nu_{m^{\prime}}\right)}
$$

It has been assumed that $u_{m}{ }^{\prime}=c / n\left(\nu_{m}{ }^{\prime}\right)$, where $n\left(v_{m}{ }^{\prime}\right)$ is the refractive index of the medium for waves of frequency $v_{m}{ }^{\prime}$. Suppose now that if $g$ is a function of order $v / c$, we have, to first order in $v / c$ :

$$
v_{m^{\prime}}=v_{0}(\mathbf{l}-g)
$$

It then follows that :

$$
\begin{gathered}
\frac{1}{n\left(\nu_{m}^{\prime}\right)}=\frac{1}{n\left(\nu_{0}-\nu_{0} g\right)}=\frac{1}{n\left(\nu_{0}\right)}-g \nu_{0}\left\{\frac{\mathrm{d}}{\mathrm{d} \nu}\left[\frac{1}{n(\nu)}\right]\right\}_{\nu \ldots} \\
=\frac{1}{n\left(\nu_{0}\right)}+\frac{g \nu_{0}}{n\left(\nu_{0}\right)^{2}}\left\{\frac{\mathrm{d} n(\nu)}{\mathrm{d} \nu}\right\}_{\nu=\nu_{0}}
\end{gathered}
$$

Substituting in (3), one finds, to first order :

$$
u_{m}=\frac{c}{n\left(v_{0}\right)}+k v
$$

where $k \equiv 1-\frac{1}{n\left(\nu_{0}\right)^{2}}+\frac{c}{v} \frac{\nu_{0}}{n\left(\nu_{0}\right)^{2}} g\left\{\frac{\mathrm{d} n(\nu)}{\mathrm{d} \nu}\right\}_{\nu=\nu_{0}}$

Here $k$ has the form of a Fresnel drag coefficient. It remains to calculate $g$.

From the expressions for the radial Doppler effect :

$$
\frac{\nu_{m}^{\prime \prime}}{v_{m^{\prime}}}=\frac{\sqrt{ }\left(1-\left[w^{\prime}\right]^{2} / c^{2}\right)}{1+w^{\prime} / u_{m}^{\prime \prime}}, \frac{\nu_{0}^{\prime \prime}}{v_{0}}=\frac{\sqrt{ }\left(1-w^{2} / c^{2}\right)}{1+w / u_{0}^{\prime \prime}}
$$

Now the rear wall of the container is at rest in $S^{\prime \prime}$ so that the light entering it is unchanged in frequency for an observer at rest in $S^{\prime \prime}$ :

$$
v_{0}^{\prime \prime}=v_{m}^{\prime \prime}
$$

Hence to first order in $v / c$ :

$$
\frac{v_{m}{ }^{\prime}}{v_{0}}=\frac{v_{0}{ }^{\prime \prime} / \nu_{0}}{v_{m}{ }^{\prime \prime} / \nu_{m}}=1+\frac{w^{\prime}}{u_{m}{ }^{\prime \prime}}-\frac{w}{u_{0}{ }^{\prime \prime}}
$$

The last two terms are correction terms and need be evaluated only to zero order, so that:

$$
w^{\prime} \doteqdot w-v, u_{m}{ }^{\prime \prime}=c / n\left(\nu_{0}{ }^{\prime \prime}\right) \risingdotseq c / n\left(\nu_{0}\right)
$$

Noting that $u_{0}{ }^{\prime \prime}=c$, one finds that equation (4) is valid, provided :

$$
g=\frac{w+(v-w) n\left(\nu_{0}\right)}{c}
$$

Combination of (5) and (7) yields an expression for the drag coefficient. One notes that $w=0$ corresponds to the case of water flowing through a tube, while $w=v$ corresponds to the moving block of glass in accordance with (2). One can say qualitatively : The effect of dispersion on the drag coefficient goes up with the disparity between the velocity of the medium and the velocity of the rear face of its container.

I am indebted to Prof. J. L. Synge for helpful correspondence.

\section{P. T. Landsberg}

Department of Applied Mathematics, University College,
Cardiff.

Synge, J. L., Relativity : The Special Theory, 142 (North-Holland : Amsterdam, 1956). 